\title{
The Cultural Influence on Perceived Quantity
}

\author{
Dong Woo Ko ${ }^{1} \&$ Dong Hyun Son ${ }^{2}$ \\ ${ }^{1}$ Department of Marketing, College of Business, Hankuk University of Foreign Studies, Korea \\ ${ }^{2}$ Department of Accounting, College of Business, Hankuk University of Foreign Studies, Korea \\ Correspondence: Dong Hyun Son, 107 Imun-Ro, Dongdaemun-Gu, Seoul, Republic of Korea. Tel: 82-2173-3172. \\ E-mail: dson@hufs.ac.kr
}

Received: February 14, 2017

Accepted: March 16, 2017

Online Published: March 20, 2017

doi:10.5430/bmr.v6n1p68

URL: https://doi.org/bmr.v6n1p68

\begin{abstract}
Variety in an assortment has been known to reduce the quantity perception of items. Individuals heavily rely on area cue when they perceive the quantity of items. People tend to perceive a single color or a shape unit as one area but the variety breaks up the areas into multiple colors or shape units. Little has been known about the mechanism underlying the relationship between the quantity perception and the variety. This research examined the cultural effects on the process of quantity perception and found that feminine cultural characteristics moderates the quantity perception when the area cues are associated with diverse colors.
\end{abstract}

Keywords: Quantity perception, Culture, Femininity

\section{Introduction}

Outside worlds are continuously reflected in inside world of people through eyes and effortlessly people perceive the information and process it to make decisions. Perception of objects are not only influenced by surroundings including texture, light, or conjunction, but people also perceived the reflected outside worlds in different ways. For example, when there is an area consisted of black and white dots, usually people incorrectly perceive that there are more black dots than white ones, which is because the black dots presumably create a salient by taking more spaces than white dots (Hoch, 2009). In addition, cultural studies suggest the different cognition of objects and background between Asian and U.S. culture (Masuda \& Nisbett, 1999); the Asian focus more on the background and the interaction between objects and its surrounding while Americans tend to have more interests only on objects. These diverse visual perception processes influence people's decision-making in various ways. Especially, recently as the development of the internet, tremendous information is delivered through webpages, mobile apps, or interactive kiosks. Depending on cultures, some countries uses a relatively monotonous design and other countries prefer to use more pictures and colors in promoting products through those medias. The emerging question, then, is if the design of variety affects the perceived quantity of items or if cultural differences influence interpretation of the perceived information, there could be unexpected influences in delivering information. Redden, and Hoch (2009) suggested that the relationship between the quantity perception and the variety. When people compare the quantity of objects consisting monotonous color and various colors, the majority believes that multi-colored sets are appeared to include more objects than a monotonous group (Redden, Hoch 2009). About such intuition, Redden, and Hoch (2009) suggested that the variety reduces the quantity perception of items. However, there are still unanswered questions especially about the cultural influences on the quantity perception process. Studying the impact of cultural influences on customers' perceptions is particularly important because previous studies have found that cultural frameworks can have significant impact of consumers' purchase behavior (Ansari \& Riasi, 2016; Riasi \& Pourmiri, 2015).

People are influenced by culture though everyday of life; we spend a lot of time on watching televisions, reading advertisements, and surfing websites, where cultures are easily reflected (Singh et al., 2004). For example, customers who live in the high level of feminine culture are exposed to more colors and pictures in their daily activities and the cultural environment affect consumers' decisions, attitudes, and information processing. What this research suggest here is the possible impact of cultural environment on consumers' quantity perception. In order to investigate the effect, this research conducted a lab experiment and found the moderating effect of culture- especially feminine feature of the culture - on the quantity perception. The rest of this paper is organized with 6 sections. Previous literature and proposed hypothesis are discussed in section 2 and 3. Section 4 describes the conducted research design and section 5 discusses the findings in this research. Finally, section 6 suggests the conclusions of the study. 


\section{Background Knowledge}

\subsection{Quantity Perception}

The term 'quantity' has been used to measure the size or volume of foods, or to estimate the number of items . Previous literature have described how people perceive the quantity of items. People have a mechanism to estimate the number of items and measure them within 17\% of differences given 100-200 milliseconds (Mandler and Shebo 1982, Piazza et al, 2004). The mechanism which helps people measure approximation is cue. For example, the area filled with items is used as a cue when people quantify a total number of items. As mentioned earlier, the visual perception of quantity is influenced by surroundings such as texture, color, and brightness. Then how does variety of items influence the perception of quantity? In fact, the majority of people that when there is the package filled with various shaped and colored items, it appears that it had more quantities than the packaged occupied with just one item because people believe that variety makes objects more easily noticed (Frick 1987, Wadhera and Capaldi-Phillips, 2014) and attract more attentions (Kolkes and Matta 2004). Redden and Hoch (2009), however, developed an interesting research question based on Gestalt psychology, which demonstrates that people perceive visual components as an organized pattern or as a whole. Because people heavily rely on the area cue, they focused whether people perceived the quantity of items as a whole or not. Also, they investigated how the variety influences the process of perceiving items. When components consist of a single color or shape, people perceived it as one area while the variety breaks up the whole. The study showed that various colors and geometric shapes reduced perceived quantity of items; they asked subjects the number of dots in the screen after showing different colored dots and shapes in a short time. The results indicated that people perceived more dots when they viewed a screen with single color dots. Also, people poured more candies when the candies had a single color. The findings can be applied to various fields which use colors to attract more customers. However, limited attention has given to the factors related to the mechanism underlying the quantity perception such as anchoring or cultural influences.

\subsection{Anchoring Effects}

Redden and Hoch (2009) proposed that the variety reduces quantity perception of items as a solid color or a shape makes it easy to connect each component of item and to consolidate into a single area. Thus, the suggested mechanism underlying the quantity perception is whether people perceive objects as a whole or not. An anchor is the psychological heuristics that influences the way people intuitively assess probabilities (Traversky, Kahneman 1974). According to the heuristic literature, people start from an implicitly suggested reference point and make adjustments to the reference point until they reach their estimation of quantity (Traversky, Kahneman 1974). For example, when a customer has a plan to buy a used car, she looks for the cars based on certain references including year, price, or mpg. In a visual study, the anchor is also one of the important terms guiding the process of perception. Bell and Bevan (1968) conducted four experiments to see how the anchor changes the perception of Gestalt organizing principles such as proximity, similarity, good continuation, and closure. In their proximity studies, when the subjects first saw a series of dots organized in seeming rows, they began to perceive more ambiguous patterns as columns. These results suggested that how anchor changes the patterns of perceptual organization in operating of Gestalt organization. The emerging question, then, is what would influence to the level of anchoring.

\subsection{Cultural Influences}

Individual responses to various aspects of the given stimulus are combined to form one reaction, which represents the perception of the given stimulus. (Marshall, Segall, Donald, Campbell, and Melville, and Herskovit, 1968) In to that extent, certain societal expressions are more likely to occur in some cultures, while different behaviors and perceptions exist in different cultures. For example, Masuda \& Nisbett (1999) examined the impact of cultural differences on the cognitive process and visual perceptions by observing the study participants' responses to the description of underwater scenes. They found that the East Asian paid more attentions to the field compared to Americans. In addition, American participants described the difference in movement between focal fishes and small fishes or animals as opposed to Japanese describing the surroundings and interactions between the surroundings and fishes.

In this research, we analyzed the cultural influences on quantity perception of items based on Hofstede's cultural dimensions. As a method of identifying differences in environmental setting, Hofstede (1984) investigated the "collective programming of the mind", which distinguished IBM employees from 50 different countries between 1967 and 1973. The study subjects were asked to respond to 32 value statements and Hofstede proposed four cultural dimensions based on the study results. First, the Individualism vs Collectivism represents the individual responsibility versus group control. Second, Power Distance stands for the dominance exerted by weak or influential society members. Third, Uncertainty Avoidance measures risk taking and lastly, Masculinity / Femininity measures 
assertive or nurturing feminine roles (Hofstede, 2004, \& ITIM, 2007). Among the four dimensions, he found that visual or color differences were associated with the femininity / masculinity. Compared to the countries with strong masculine culture, those with feminine culture have conveyed more aesthetic details (Singh et al. 2004). Earlier cultural studies showed that there were significantly different cultural depiction between American and Japanese automotive, electronics, and retail websites (Singh et al. 2002). Japanese websites scored significantly higher in the femininity cultural dimension compared to American websites. Japanese websites used more colors, bold colors, and more images. These characteristics were also found in other countries with high femininity, including Mexico (Singh et al. 2004), China, India (Singh et al. 2005), and South Korea (Ko ,Seo, Jung 2013). The results suggest that individuals in highly feminine cultures have been more exposed to the variety of colors and shapes, leading to develop a high anchor toward the number of colors.

The Masuda \& Nisbett (1999)' study discussed earlier suggest that the history can provide a possible explanation of this difference between the two cultural regions. Farming has been a primary source of earning for Chinese as opposed to keeping animal and fishing for the Western. Compared to keep animals, cooperation with other social members has been regarded as an important societal virtue in farming environments that lead eastern culture to be less sensitive about the variety. Alternatively, the different sense of variety between cultures can be extended to visual perception and anchor of varieties. If Japanese were more exposed to various colors and shapes, Japanese might have a high reference about color and shape. As a result, Japanese would be less sensitive about perceiving quantity of items with various colors and shapes. This argument also indicates that it would be possible to make individuals perceive less quantity by priming femininity cultural characteristics.

H1. The femininity cultural characteristics are moderated when individuals are exposed to more variety of colors

H2a. The femininity cultural aspect helps individuals perceive more quantity from the object with various colors and shapes compared to the object with a solid color and shape

$\mathrm{H} 2 \mathrm{~b}$. The masculinity cultural aspect helps individuals perceive less quantity from the object with various colors and shapes than the object consisting of monotonous component

\section{Purpose of Research}

Redden and Hoch (2009) suggested that people's quantity perception decreased when the objects were varied. Gestalt's organizing theory supports their findings that single- colored or shaped units help people perceive the object as a single unit or as a whole. In this research, we examined whether different anchoring colors differentiate the quantity perception. Also, feminine cultural characteristics were primed to increase the referred number of color or shapes. Because the heuristic, anchor, is a reference point to reach their estimation, increased feminine cultural characteristics help people perceive a variety of items as a whole or disturb the color difference to break up the perception of object. If individuals do not have a difficulty connecting the variety of of colors, they perceive more quantity in that more attentions are given to varied set and the misattribute attention influences quantity perception process (Flokes \& Matta, 2004).

\section{Method}

\subsection{Study}

In order to test the proposed hypotheses, we designed an experiment based on Redden and Hoch (2009)' study. We investigated how priming of femininity cultural characteristics affect perceived quantity of items by comparing the variety primed group and monotonous primed group.

\subsection{Experimental Manipulation and Procedure}

We recruited eighty-five participants from one university in South Korea. They were seated in front of computers and randomly assigned into the variety primed groupand the monotonous primed group. For the variety primed group, ten different pictures containing various shapes and colors were presented to forty students for 750 milliseconds three times, totaling 30 pictures per subject. This process is intended to prime the femininity features. In order to prevent the order effects, different series of pictures were used in the second and the third sets of presentation. The pictures were presented on a 21 inch LCE monitor located in an experiment lab, approximately $100 \mathrm{~cm}$ apart from the participants. Subjects were guided to comfortably look at the pictures on the screen and to keep their attentions to the fixation cross in the center of monitors. For the monotonous primed group, 45 students were presented the same pictures as the ones given to the variety primed group, except for the color. The colors in the pictures were switched to black and white, using picture editing program (see Appendix 1). 
After priming femininity characteristics, two questions were asked to both groups for the manipulation check; femininity cultural characteristics and the information of pictures. Four pictures including one picture that was not presented to them earlier were given to participants. Then, participants were asked to recall the pictures and to choose the picture that was not presented. Also, the individual level of femininity characteristic was measured based on previous literature (Yoo \& Donthu, 2002). After the manipulation process, both groups were asked to estimate the percentage of six randomly presented items - three with various colored dots (variety dots) and remaining three with blackdots (black dots). The patterns were created based on a $3 \mathrm{X} 2$ full factorial design.

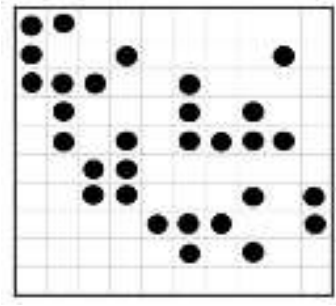

$30 \%$ Black

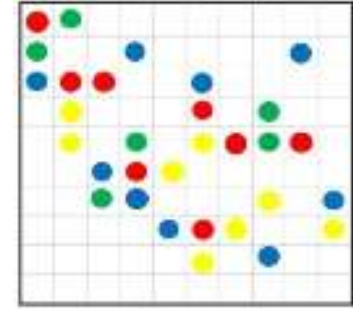

$30 \%$ Variety

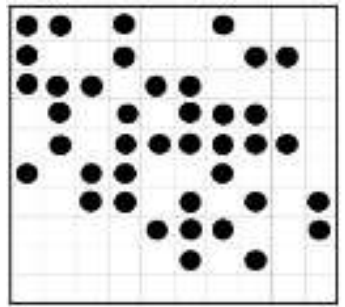

$40 \%$ Black

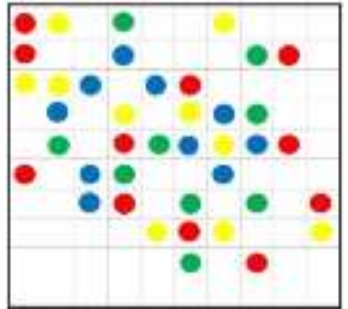

$40 \%$ Variety

Figure 1. Sample Stimuli

For the variety, three different percentages $(30 \%, 40 \%, 50 \%)$ and two varieties (variety dots, black dots) consist the factorial cells. The patterns of stimuli were designed based on $10 \times 10$ squared matrix. The 5.5-inched matrixes were presented on the center of the screen with black outside boarders and light inside boarders, which divided the square into 100 individual squares (see Figure 1). The subjects were allowed to see each item for 750 milliseconds and then asked to estimate the percentage of area filled with dots in each matrix. The study was done with debriefing.

\section{Results}

A total of 85 students (mean age 24.3 years, $61 \%$ males) voluntarily participated in this study and candy bars were provided to compensate for their participation. In order to investigate the priming effect of the variety on the quantity perception, a repeated measure of ANOVA was employed with the various percentages filled - 30, 40, and 50 percentages of matrix - as a within-subject factor. The AVOVA test suggested that the main effect of the percentages filled on matrix was significant $(\mathrm{F}(1,46)=92.66, \mathrm{p}<.01)$ and the interaction effect between variety priming $($ variety primig VS. monotonous priming) and estimation of items (variety dots VS. Black dots) $)(\mathrm{F}(1,46)=4.57, \mathrm{p}<.05)$. The results indicate that as people are more exposed to a variety of colors, they are more likely to perceive a large quantity of items (See Figure 2). Also, after priming the variety, the variety primed group showed the high level of femininity cultural characteristics compared to the monotonous primed group $(\mathrm{t}(46)=1.94, \mathrm{p}<.1)$, suggesting that both groups were primed well, and priming colors influence the individual level of feminine cultural characteristics.

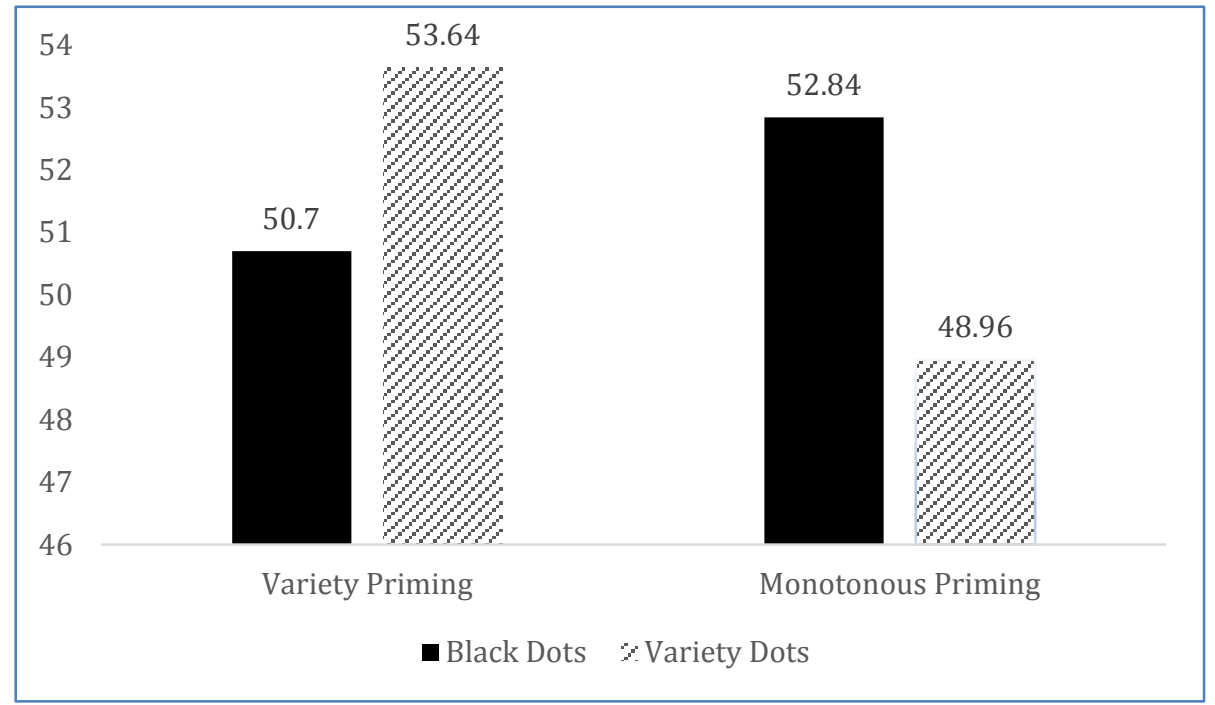

Figure 2. The Effect of Color Priming on Quantity Perception 
In this study, we hypothesized that the high level of feminine cultural feature increases the perceived quantity from variety setting. To examine the expected cultural effect on the quantity perception of items, the hierarchical linear model (HLM; Raudenbush and Bryk, 2002) was used to investigate the interaction effect between the individual-level and the group-level effects of cultural characteristics (variety VS. black dots) on the quantity perception. In this study, the quantity perception at the individual level was nested within the different variety priming and the estimation of items group. The femininity, as a cultural characteristic, was an independent variable and gender and age were control variables. At the group level, color priming (variety vs. monotonous priming) and the estimation of items (variety VS. black dots) were employed.

$$
\text { Quantity Perception }=\beta_{0 j}+\beta_{l j} \text { Femininity }_{i j}+\beta_{2} \text { Age }_{i j}+\beta_{3} \text { Gender }_{i j}+\varepsilon_{i j} \text {, }
$$

where $i, i=1,2, \ldots \ldots \ldots . .85$ and variety primed and estimation of items group $j, j=1,2,3,4$,

To investigate heterogeneity in the individual level effect across the four variety conditions, we tested the intercept parameter, $\beta_{0 j}$, and the coefficient of femininity, $\beta_{l j}$, varying across four level 2 conditions.

$$
\beta_{0 j}=\gamma_{0 j}+\mu_{0 j} \text {, }
$$

$$
\beta_{l j}=\gamma_{l j}+\mu_{l j}
$$

We estimated HLM with maximum likelihood algorithm. The series of results from simplest benchmark model with only level 1 to the most complex analysis of proposed model were presented in table 1 . The comparison of benchmark and proposed model showed the outperformance of proposed model $\left(\mathrm{BIC}_{\text {benchmark }}=3090.69 \mathrm{vs}\right.$. $\mathrm{BIC}_{\text {proposed }}$ $=3074.83$ ).

The parameter estimation of thee-way interaction among estimation of items, variety priming and femininity show the marginally significant effect on quantity perception $(\mathrm{r}=3.04, \mathrm{t}=2.85, \mathrm{p}<.1)$. The results indicate that individuals having high level of femininity cultural characteristics and exposed to more colors, they are more likely to perceive larger quantity from more color variety conditions. These results support the proposed $\mathrm{H} 2 \mathrm{a}$ and $\mathrm{H} 2 \mathrm{~b}$.

Table 1. The Effect of Culture on Quantity Perception

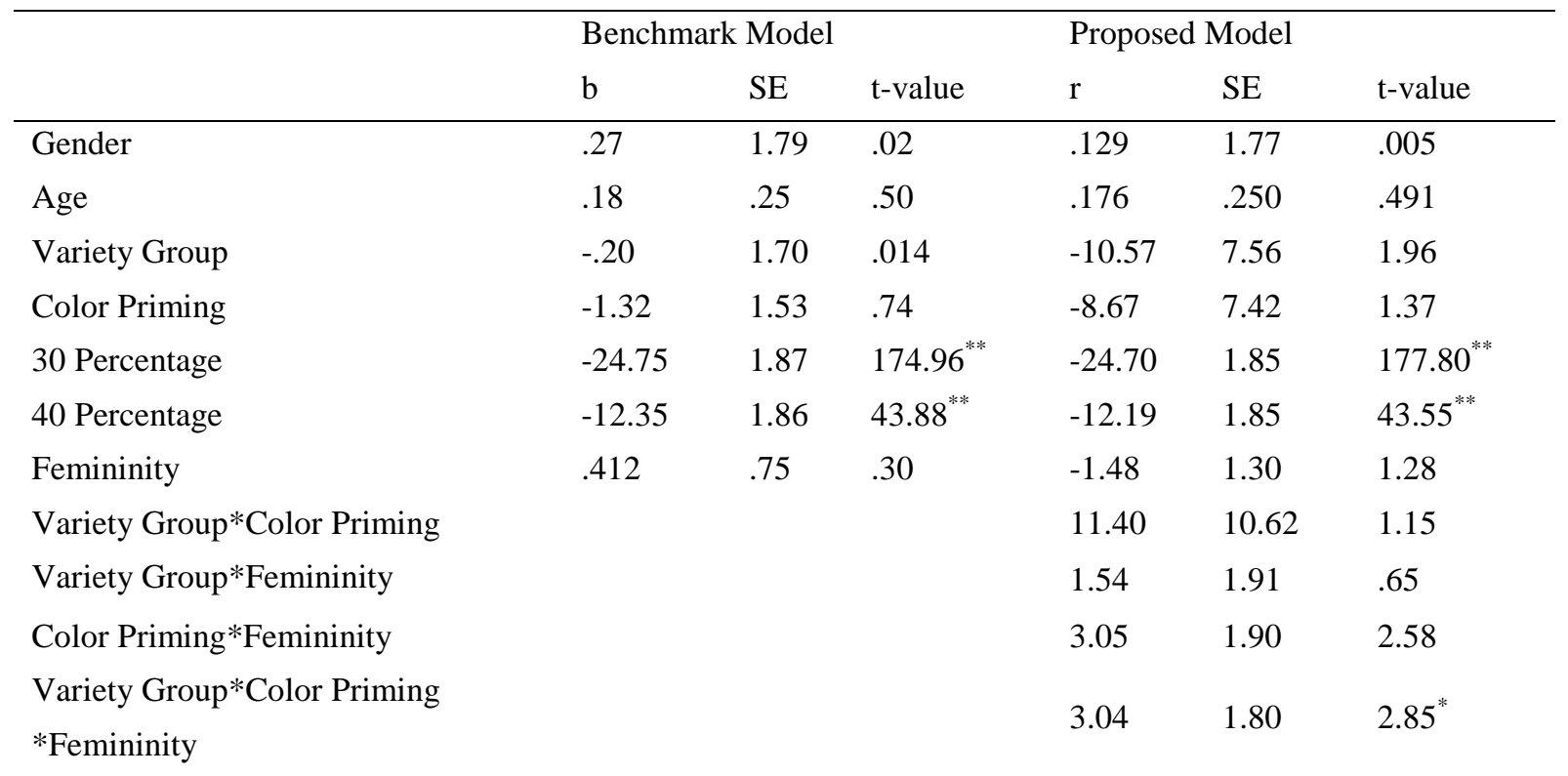

This research is designed to investigate how cultural environment influences consumers' cultural characteristics and its' influences on consumers' quantity perception. As predicted, The ANOVA analysis showed that as people are exposed to variety of colors and shapes, they perceived larger quantities from the item with various colors than monotonous color. Also the HLM analysis indicated that these patterns are significant as femininity cultural characteristic is salient.

As previous literature suggested, consumers are more comfortable to estimate the quantity of item when it is consist of monotonous components compared to various components by operating Gestalt organization(perceived it as a whole); however, frequent exposure to the variety conditions stimulated feminine cultural characteristics and made them perceive more quantity of items, which are filled with various colored dots. The results suggest that priming of femininity made consumers feel comfortable in organizing items with various dots. 
These patterns of perception process indicated that the external environment and cultural characteristics are important factors in understanding consumers' perception process.

\section{General Discussion}

Previous literature on culture has focused to identify the reflected culture in our daily life such as design of website (Ko et al, 2013), mobile apps (Lim et al, 2014) and advertising (Singh et al, 2004) and to understand its influences on consumers' behavioral areas such as attitude and WTP. However, limited attention has given to more fundamental area, the effect of culture on perception process. In fact, with the development of internet and mobile technology, consumers frequently access culturally customized online websites and mobile apps to get information and share information with other social members. Korean online travel agencies, for example, use more pictures and colors to promote their products while U.S. online travel agencies use more texts to deliver detailed product information (Ko et al, 2013). Thus, people who live in Korea naturally exposed more often to the variety of colors and shapes compared to consumers in U.S.. The findings of this research suggest that the culturally customized internet environments influence consumers' perception process; the high exposure to the feminine features influenced the changes of individual level of cultural characters and quantity perception of items.

The findings of this research also contributes to the literature on visual perception and cultural studies. In spite of interesting finding in visual perception studies, limited study has shown the cultural influences. This research provides one possible intuition to understand the cultural influence on visual perception process and extended the finding in Gestalt's psychology.

In spite of the interesting findings in this research, there is a limitation that we have to discuss. This research collected date from a single culture to test whether the color priming influences individual level of cultural characteristics within a culture. A prominent feature of culture is that the culture is learned process. Each individual carries a specific pattern of thinking, feeling, and behaving based on what they intentionally or unintentionally have learned from the social environment. This is the main reasons why individuals that live in same area share a similar patterns of behavior and why individuals adapt themselves to new environment. This research empirically examined whether individual level of cultural characteristics varied when they were continuously exposed to cultural features by collecting the data from limited a single culture and by excluding the external validity. However, it would be interesting to extend the current findings by comparing with other cultures.

\section{Acknowledgement}

This research is supported by Hankuk University of Foreign Studies Research Fund.

\section{References}

Ansari, A., \& Riasi, A. (2016). An investigation of factors affecting brand advertising success and effectiveness. International Business Research, 9(4), 20 - 30. https://doi.org/10.5539/ibr.v9n4p20

Bell, R. A., \& Bevan, W. (1968). Influence of anchors upon the operation of certain Gestalt organising principles. Journal of Experimental Psychology, 78, 670 - 678. https://doi.org/10.1037/h0026616

Broniarczyk, Susan M., Hoyer. W.D., \& McAlister, L. (1998). Consumers' Perceptions of the Assortment Offered in a Grocery Category: The Impact of Item Reduction. Journal of Marketing Research, 35(2), 166-76. https://doi.org/10.2307/3151845

Choi, I., Nisbett, R. E., \& Norenzayan, A. (1999). Causal attribution across cultures: Variation and universality. Psychological Bulletin, 125, 47-63. https://doi.org/10.1037/0033-2909.125.1.47

Folkes, V., \& Matta, S. (2004). The Effect of Package Shape on Consumers' Judgments of Product Volume: Attention as a Mental Contaminant. Journal of Consumer Research, 31(2), 390-401. https://doi.org/10.1086/422117

Hofstede, G. (1984). Cultural dimensions in management and planning. Asia Pacific Journal of Management, 1(2), 81-99. https://doi.org/10.1007/BF01733682

Hofstede, G., \& Hofstede, G. V. (2004). Cultures and organizations: software for the mind. New York: McGraw-Hill.

Lim, S. D., Bently, P., Kanakam, N., Ishikawa, F., \& Honiden, S. (2014). Investigating Country Differences in Mobile App User Behavior and Challenges for Software Engineering. IEEE Transactions on Software Engineering, 5589(n), 1-1.

Mast, M. S., Sieverding, M., Esslen, M., Grader, K., \& Jancke, L. (2008). Masculinity causes speeding in young men. Accident Analysis and Prevention, 40(2), 840-842. https://doi.org/10.1016/j.aap.2007.09.028 
Mandler, G., \& Shebo, B. J. (1982). Subitizing: An Analysis of Its Component Processes. Journal of Experimental Psychology: General, 111(1), 1-22. https://doi.org/10.1037/0096-3445.111.1.1

Norenzayan, A., \& Nisbett, R. E. (2000). Culture and Causal Cognition. Current Direction in Psychological Science, 9(4), 132-135. https://doi.org/10.1111/1467-8721.00077

Piazza, M., Pinel, P., Le Bihan, D., \& Dehaene, S. (2007). A magnitude code common to numerosities and number symbols in human intraparietal cortex. Neuron, 53, 293-305. https://doi.org/10.1016/j.neuron.2006.11.022

Redden, J. P., and Hoch, J. H. (2009). The presence of variety reduces perceived quantity. Journal of consumer research, 36. https://doi.org/10.1086/598971

Riasi, A., \& Pourmiri, S. (2015). Effects of online marketing on Iranian ecotourism industry: Economic, sociological, and cultural aspects. Management Science Letters, 5(10), 915-926. https://doi.org/10.5267/j.msl.2015.8.005

Ruan, D., Chen, G., Kerre, E. E., \& Wets, G. (2005). Intelligent data mining, Heideberg: Springer.

Singh, N., \& Baack, D. W. (2004). Web site adaptation: A cross-cultural comparison of U.S. and Mexican Web sites. Journal of Computer-Mediated Communication, 9(4). https://doi.org/10.1111/j.1083-6101.2004.tb00298.x

Singh, N., Zhao, J., \& Hu, X. (2005). Analyzing cultural information on web sites: A cross-national study of web site from China, India, Japan, and the U.S.. International Marketing Review, 22(2). https://doi.org/10.1108/02651330510593241

Tversky, A., \& Kahneman, D. (1974). Judgement under uncertainty: Heuristics and biases. Science, 185, 1124-1130. https://doi.org/10.1126/science.185.4157.1124

Treisman, AM., Gelade, G. (1980). A feature-interation theory of attention. Cognitive Psychology, 12. 97-136. https://doi.org/10.1016/0010-0285(80)90005-5

Wadhera, D., \& Capaldi-Phillips, E. C. (2014). A review of visual cues associated with food on food acceptance and consumption. Eating Behaviors, 15(1), 132-143. https://doi.org/10.1016/j.eatbeh.2013.11.003

Yoo, B., \& Donthu, N. (2002). The effects of marketing education and individual cultural values on marketing ethics of students. Journal of Marketing Education, 24(2), 92-103. https://doi.org/10.1177/0273475302242002 
Appendix 1. Pictures for the variety priming

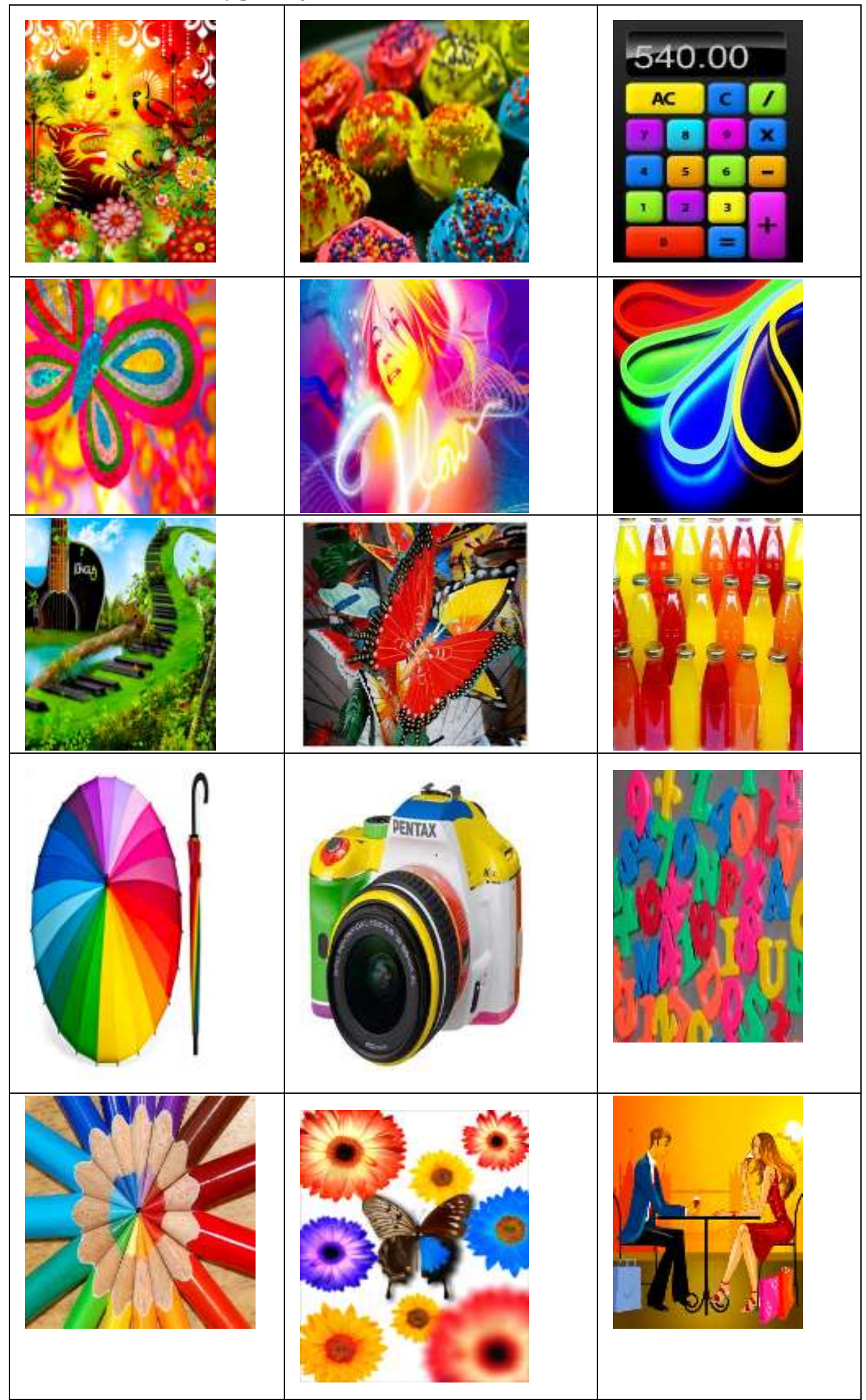


Appendix 1. Pictures for monotonous priming

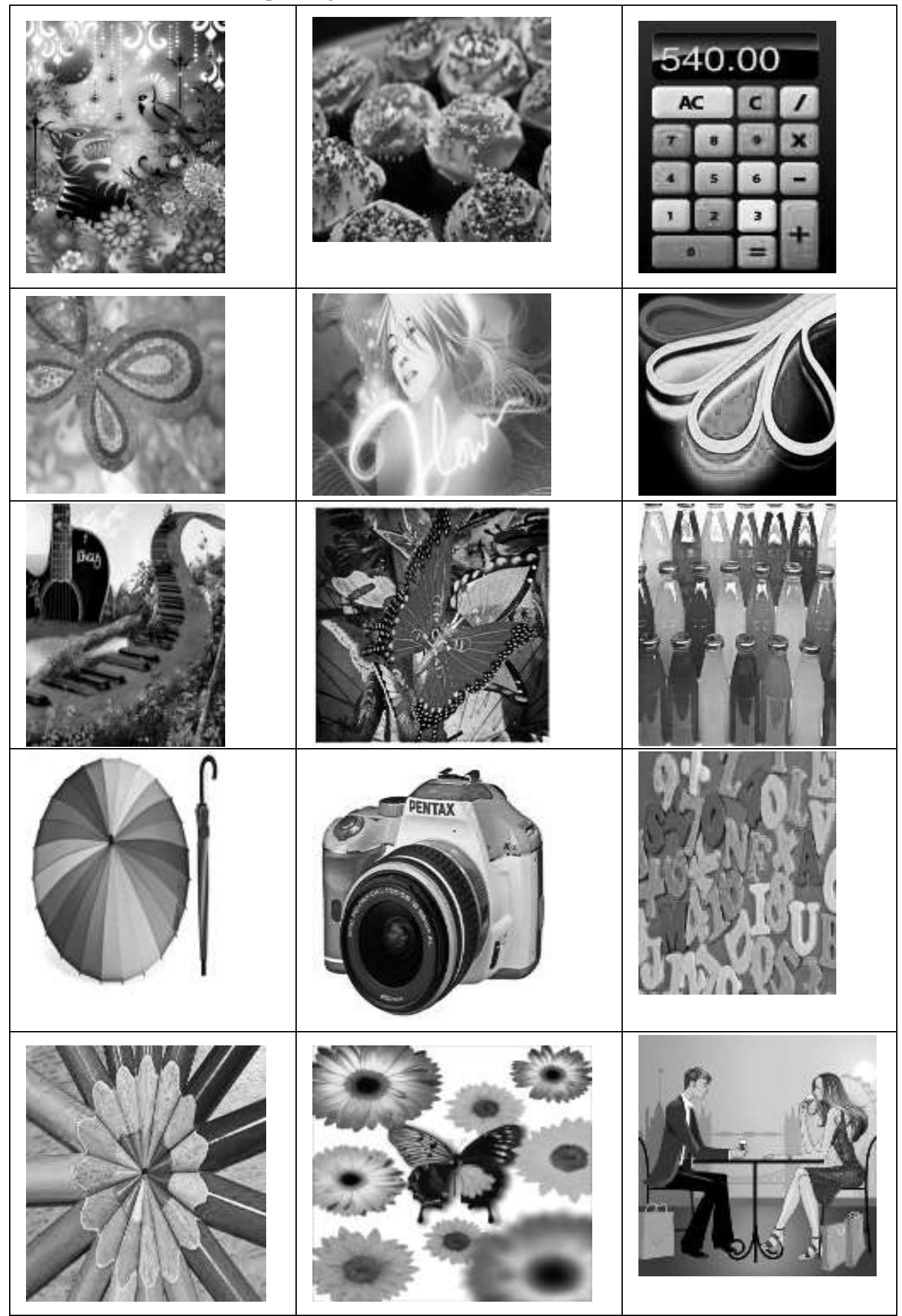

